\title{
Research on Route Choice of Passengers Based on The Travel Time
}

\author{
Wang Qi * \\ Beijing JiaoTong University \\ School of Traffic and Transportation \\ Beijing, China \\ wangqibjtu@163.com \\ * Corresponding Author
}

\author{
Ma Kun \\ Beijing JiaoTong University \\ School of Traffic and Transportation \\ Beijing, China \\ makunsy@sina.com
}

\begin{abstract}
With the development of networked urban rail transit system, The ticket income distribution between each operator and line is becoming more and more complex. To accurately grasp the trip characteristics of passengers who choose the rail transit as their trip mode is of vital importance. The related information about the time and space can be obtained from Automated Fare Collection system of the urban rail transit. And to summarize the characteristics of the distribution of passengers travel time. By analyzing the data obtained. Calculate the proportion of passengers on different routes of the Origin-Destination and construct models about the passengers' route choices, based on the time distribution of the passengers in a period of time about one Origin-Destination. Select a practical case of Beijing Rail Transit Network to validate the correctness and accuracy of the model by the methods of simulation or the actual data of Automated Fare Collection system.
\end{abstract}

Keywords-Rail Transit; Travel Time; Pedestrian Flow; Urban Rail Transit; Ticket Revenue Clearing

\section{INTRODUCTION}

A large scale of urban rain transit network has been established in many cities, thus as Beijing, Shanghai, Guangzhou, Entering the network operation stage. The "one-ticket transfer" mode has applied to Rail transit network operators. The mode can improve the convenience of the passengers and save the transfer time. However, the transfer information of passengers is difficult to accurately obtain, therefore it's difficult to recognize the passenger's travel route. The accurately analysis of passenger route choice behavior provides a thorough grounding in the fare clearing, and basic operations planning and traffic related work. Si Bingfeng ${ }^{1]}$ constructed the generalized cost function of urban rail transit network, and had analyzed the route choice behavior of passengers in urban rail transit network. Luo Qin ${ }^{[2]}$ constructed the theoretical framework of the passenger flow distribution based on the basic characteristics of network operations, and studied simulation system about the distribution of networked passengers flow. As the travel time of different travel routes in the network are different. The paper studies route choice behavior by comparing or classifying the different passengers' travel time.

In this paper, a passenger route reckoning model is constructed, by analyzing the rules of passenger travel time through the data in Automated Fare Collection (AFC) system and identifying of travel route choice behavior of passengers. Finally, the results are validated by the methods of simulation and the actual data (about a practical Origin-Destination in Beijing).

\section{THE PROBLEM OF THE RAIL TRANSIT} PASSENGER ROUTE CHOICE

At present, the Rail transit networked operation has been inducing will cause the difference of the passengers travel route choice behavior. Travel time influences most on the travel route choice behavior of passengers ${ }^{[1,3]}$. In general, the most important factor to determine the route of the passengers chosen is travel time. By means of the AFC system, the travel time of each passenger can be obtained by calculating. To analyze the route choice behavior of passengers based on the distribution of passenger travel time.

\section{A. The Relationship between the Route Choice and Travel Time}

From the statistics, it analyzed the form or change of the travel time which has studied the problem of the passengers' route choice through the passenger travel time data recorded in the AFC system. In some OD pairs, as Fig.2-1, From Gongzhufen to Chaoyangmen, the curve has two peaks in the travel time. The OD pair performs the distribution with two peaks approximately. Comparing with the practical network, there are some differences in the travel time of the two routes. Therefore, there are two effective routes so the travel time is required to be different.

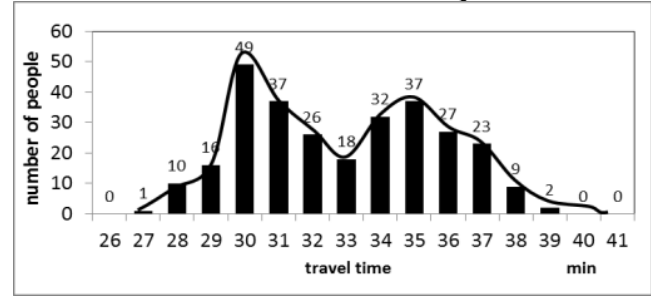

Figure 2-1. The Distribution Dagram of Gongzhufen - Chaoyangmen OD Travel Time

If the travel time distribution function of the two routes of one OD appears to be the same (or similar) situation, on this occasion, the result of calculation is invalid, because 
the essence of the method is to calculate the proportion of passenger flow by the different distribution of travel time. In this case, there is no difference between the two routes from the aspect of travel time. Passenger's choice will have a strong random in this case, it's believed that the proportion of passenger flow of the two routes is equal.

It isn't entirely consistent of the passengers' route choices in the several effective routes. So, research on the characteristics of passenger travel time and the relationship between the travel time and travel distance or transfer times through dividing of travel time, according to the characteristics of the travel time, analyzing the characteristics of the passengers' route choices ${ }^{[4,5]}$.

\section{B. The Composition of Passengers' Travel Time}

The Time-record of passengers travel time based on AFC data is the time from passengers at the Origin metro station swipe card in bound to passengers at the Destination metro station swipe card outbound, which does not include the time of passengers entering the rail transit station, buying ticket, security and so on.

In two cases such including without transfer and transfer, Part of the time is fixed, and the other part of the time will show different because of the characteristics of the passenger's personal or other factors. In this paper, these two kinds of time are divided into two categories: Travel ESSential time and Travel RANdom time.

So the travel time can be expressed as:

$$
T=t_{\text {ess }}+t_{\text {ran }}
$$

So, passengers' travel time of one OD is divided into two parts:

A part is essential time which includes OTT and the necessary part of ACT, PWT, ICT, IWT, EGT; the other part is random time which includes the time of Import and Export station with more than ESS time, the summation of the time of waiting for the train, the time of Transfer walking time with more than ESS time.

\section{PASSENGERS' ROUTE RECKONING MODEL}

\section{A. Probability Density Function of Travel Time}

The essential Time can be considered as the same for different passengers on fixed route, therefore, the ESS time of that route is fixed time for the fixed OD. The difference is caused by a variety of conditions at random times. In the actual situation, the random time has a constant maximum value (Exclusion of few special cases). Therefore, there is a upper bound for random time. Assuming the maximum value of the random time is $T_{e s s}^{\max }$, So the density function of $T_{r a n}$ can be expressed as:

$$
\varphi_{\text {ran }}(t)=\left\{\begin{array}{cc}
f(t), & 0<t<T_{\text {ran }}^{\max } \\
0, & \text { else }
\end{array}\right.
$$

According to the formula (3-1), the density function of the passenger travel time $\mathrm{T}$ is:

$$
\varphi_{T}(t)=\left\{\begin{array}{cc}
f\left(t-T_{\text {ess }}\right), & T_{\text {ess }}<t<T_{\text {ess }}+T_{\text {ran }}^{\max } \\
0, & \text { else }
\end{array}\right.
$$

According to the formula (3-1), (3-2), obviously, $\varphi_{\text {ran }}(t)$ is a Deformation function of the Independent variable has been parallel shift with $\varphi_{T}(t)$. The passengers' travel time (T) obey the distribution of the function $\varphi_{T}(t)$.
For each route with a fixed OD, the composition of random time:

$$
T_{s j}=\Delta t_{a c t}+\Delta t_{p w t}+\Delta t_{i c t}+\Delta t_{i w t}+\Delta t_{e g t}
$$

$\Delta t_{\text {act }}, \Delta t_{i c t}, \Delta t_{\text {egt }}$ can be considered as equidistribution.

$\Delta t_{p w t}$ is platform waiting time. $\Delta t_{i w t}$ is Transfer waiting time, which is a part of the Platform Wait Time. $\Delta t_{p w t}$ is considered the waiting time of that is not transfer, That is to say, it's the waiting time of the first train. $\Delta t_{i w t}$ is the waiting time about the several trains later.

In the peak period of operation, a great number of people are on the platform. Generally, the transport capacity of the train is not to satisfy demand, the remaining capacity is insufficient, In this situation, It is a part of the waiting passengers have no alternative other than waiting the next train, the site is called "left behind".

Based on spot investigation in Beijing rail transit network, the "left behind" appears in most stations in the network in different degree in the morning peak period ${ }^{[6]}$. Because of the platform is crowded, disorder of boarding, A lot of vehicle doors.it is not appeared that it got it as soon as someone reach here. The passengers of arriving at first is pretty randomness, who are not waiting uniformly at the entrance to the vehicle entrance, they will gather at the door when the train is about to enter the station. So the boarding is in strong randomicity. According to spot investigation, there are few passengers need more than two times "left behind". Therefore, $\Delta t_{p w t}, \Delta t_{i w t}$ need to increase $T_{f}$ or $2 T_{f}$.

$$
\begin{cases}\Delta t_{p w t}=\Delta t_{p w t}{ }^{\prime}+i T_{f} & , \quad(i=1,2) \\ \Delta t_{i w t}=\Delta t_{i w t}{ }^{\prime}+i T_{f} & , \quad(i=1,2)\end{cases}
$$

According to sum up, these random Times can be considered as uniform distribution. So the density function of $T_{r a n}$ is:

$$
\varphi_{\text {ran }}(t)=\varphi_{1}(t) \times \varphi_{1}(t) \times \square \square \square \times \varphi_{1}(t)
$$

\section{B. Multi-Route Proportional Model}

For a fixed OD, the effective routes can be chosen by passengers is usually more than one ${ }^{[7]}$. The percentage of passengers choosing each effective route is recorded as $\mu_{1}$, $\mu_{2}$, - $-\mu_{n}$, and $\sum \mu_{n}=1$. By formula (3-5), the travel time distribution of all the passengers with the fixed OD is:

$$
\varphi_{T o d}(t)=\sum_{i=1}^{n} \mu_{i} \varphi_{T i}(t)
$$

\section{The calculation method for two effective routes}

Assuming that the route with a fixed OD is more than two, passengers can directly evaluate the removal of the worse route, the route is selected in the best route $L_{1}$ and the suboptimal route $L_{2}$.

Assuming $\varphi_{L 1}(t)$ is the density function with Passenger travel time of route $L_{1}$, which is not Zero in $\left[\sigma_{a}, \sigma_{A}\right]$, In other areas is zero. 
$\varphi_{L 2}(t)$ is the density function with Passenger travel time of route $L_{2}$,which is not Zero with $\left[\sigma_{b}, \sigma_{B}\right]$, In other areas are Zero. Therefore, $\sigma_{a}$ is essential time of $L_{1}, \sigma_{b}$ is essential time of $L_{2}$.

Suppose a fixed time in one day, the proportion of the two routes are $\mu_{1}, \mu_{2}=1-\mu_{1}$.So, by formula (3-6), the density function with Passenger travel time of two routes is $\left[\sigma_{a}, \sigma_{A}\right]$.

$$
\varphi_{T o d}(t)=\mu_{1} \varphi_{T 1}(t)+\left(1-\mu_{1}\right) \varphi_{T 2}(t)
$$

The route selection problem is solved through AFC data in a fixed OD for a period of time. So T within travel time which is $[m, n]$, at the same time to require the scope to meet:

$$
\left\{\begin{array}{l}
\min \left\{\sigma_{a}, \sigma_{b}\right\}<m<\min \left\{\sigma_{A}, \sigma_{B}\right\} \\
\max \left\{\sigma_{a}, \sigma_{b}\right\}<n<\max \left\{\sigma_{A}, \sigma_{B}\right\}
\end{array}\right.
$$

The probability $\left(P_{m n}\right)$ that one passenger's travel time is in the interval $[m, n]$.

$$
\begin{aligned}
P_{m n} & =\int_{m}^{n}\left(\mu_{1} \varphi_{T 1}(t)+\left(1-\mu_{1}\right) \varphi_{T 2}(t)\right) d t \\
& =\mu_{1} \int_{m}^{n} \varphi_{T 1}(t) d t+\left(1-\mu_{1}\right) \int_{m}^{n} \varphi_{T 2}(t) d t
\end{aligned}
$$

In a period of time, by AFC data, assuming the Number of passengers is $C$ in the fixed OD. Travel time is within the range $[m, n]$ with the passengers is $R_{m n}$, so it is:

$$
P_{m n}=\frac{R_{m n}}{C}
$$

Through the AFC system data collation, it attained $R_{m n}$ and $C$, then calculate the $P_{m n}$. Then it Calculate $\mu_{1}$ by formula (3-9) and formula (3-10). That is, the probability of the passengers to choose the two route.

\section{The calculation method with three effective routes}

Generally, The effective route is no more than three when passengers choose the route with the fixed OD ${ }^{[8,9]}$. Assume that the route between a fixed OD is more than three. But passengers can directly evaluate the removal of the worse route, Selection on $L_{1} 、 L_{2} 、 L_{3}$ routes. The calculation method of the three effective routes is same as the calculation method of the two effective routes.

\section{CASE STUding}

\section{A. The basic information}

The Dawanglu station to Yonghe Temple station is chosen in this paper. According to the analysis in chapter 3 , the passengers can directly evaluate and removal the worse route, selecting by the optimal and sub-optimal routes. There are two valid routes,

Route 1: Dawanglu - Jianguomen (line 2) - Yonghe Temple.

Route 2: Dawanglu - Dongdan (line 5) - Yonghe Temple.

The essential time includes the time of the train running between station and the time of the stop, and the essential time for the transfer. Therefore, the essential time of route 1 is $\sigma_{a}=18 \mathrm{~min}$, the essential time of route 2 is $\sigma_{a}=20 \mathrm{~min}$.

\section{B. Simulation Validation}

According to the spot investigation, Line 1 departure interval recorded as $2 \mathrm{~min}$, Line 2 departure interval recorded as $2 \mathrm{~min}$, Line 5 departure interval recorded as $2 \mathrm{~min}$. In Dongdan station, the average transfer time is 356 seconds $(6 \mathrm{~min})$ from line 1 to line 5 . In Jianguomen station, the average transfer time is 130 seconds $(2 \mathrm{~min})$ from line 1 to line 2 .

Assuming 200 passengers travel by route 1,100 passengers travel by route 2 . The essential time for each passenger is determined by the route. The travel time period of 200 passengers about route 1 is superimposed to the travel time distribution of the route 1 with 200 passengers. The travel time period of 100 passengers about route 2 is superimposed to the travel time distribution of the route 2 with 100 passengers.

Fig.4-1 is a normal distribution diagram by fitting of random numbers with produced.

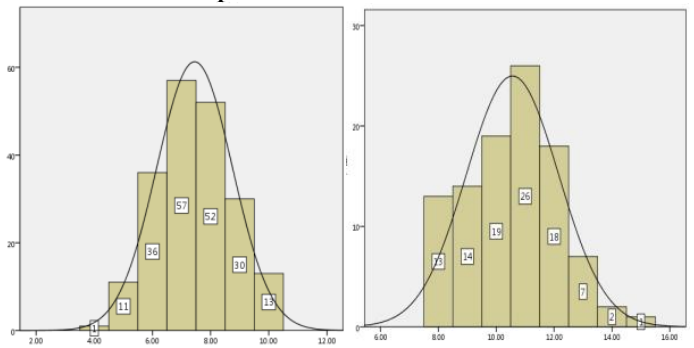

Figure 4-1. The Fitting Diagram of Passenger Random Time Normal Function about Route 1,2

The essential time of route $1 \sigma_{a}=18 \mathrm{~min}$, the essential time of route $2 \sigma_{a}=20 \mathrm{~min}$. Therefore the random time distribution of Route 2 needs to add the essential time difference between the two routes, which is $\sigma_{b}-\sigma_{a}=2 \mathrm{~min}$. The total time distribution is calculated, as shown in fig.42.

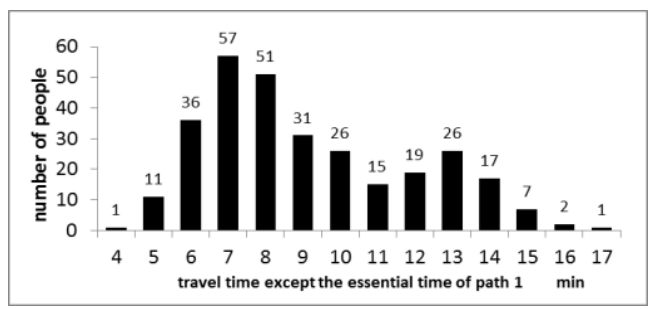

Figure 4-2. The Distribution Diagram of Passenger Total Travel Time

According to the third chapter, $\mu_{1}=0.711$.

So, the probability of choosing the route 1 is $71.1 \%$, and the number of passengers is 213 ; the probability of choosing the route 2 is $28.9 \%$, and the number is 87 . Deviation is $4.33 \%$.

The essential time of the two routes has a nonsignificant effect on the results. But their difference would have a big impact. Table 4-1 shows the result of 15 consecutive simulations for $\mathrm{T} 2=\mathrm{T} 1+2$.

At the same time, in order to analyze the influence of the difference between the two routes. $\mathrm{T} 2=\mathrm{T} 1+4$ was also simulated 15 consecutive simulations. To the actual situations, the two cases are corresponding to the peak 
period $(\mathrm{T} 2=\mathrm{T} 1+2)$ and the daily time $(\mathrm{T} 2=\mathrm{T} 1+4)$. In these 30 simulations, the number of passengers is about 300 and 100. The proportion of two routes is also changing.

Passenger Flow clearing is based on The travel time distribution for all passengers and the random time density function of two routes calculating the number of passengers on the two routes ${ }^{[10]}$. The last line is the sum of these 15 results in Table 4-1. The total deviation is smaller than before. According to the clearing results, the accuracy of this method is demonstrated.

TABLE 4-1 VALIDATION RESULTS

\begin{tabular}{|c|c|c|c|c|c|c|c|c|c|c|c|}
\hline \multicolumn{7}{|c|}{$\mathrm{T} 2=\mathrm{T} 1+2$} & \multicolumn{6}{|c|}{$\mathrm{T}=\mathrm{T}+4$} \\
\hline No & $\begin{array}{c}\text { Number of } \\
\text { people }\end{array}$ & $\begin{array}{c}\text { Verification } \\
\text { results }\end{array}$ & $\begin{array}{c}\text { Devia } \\
\text { tion }\end{array}$ & No & $\begin{array}{c}\text { Number of } \\
\text { people }\end{array}$ & $\begin{array}{c}\text { Verification } \\
\text { results }\end{array}$ & $\begin{array}{c}\text { Devia } \\
\text { tion }\end{array}$ \\
\hline 1 & 200 & 100 & 213 & 87 & $4.33 \%$ & 1 & 200 & 100 & 192 & 108 & $2.67 \%$ \\
\hline 2 & 80 & 19 & 82 & 17 & $2.02 \%$ & 2 & 86 & 25 & 87 & 24 & $0.90 \%$ \\
\hline 3 & 234 & 89 & 249 & 74 & $4.64 \%$ & 3 & 224 & 75 & 232 & 67 & $2.68 \%$ \\
\hline 4 & 258 & 61 & 271 & 48 & $4.08 \%$ & 4 & 260 & 59 & 259 & 60 & $0.31 \%$ \\
\hline 5 & 244 & 39 & 230 & 53 & $4.95 \%$ & 5 & 241 & 42 & 228 & 55 & $4.59 \%$ \\
\hline 6 & 128 & 151 & 126 & 153 & $0.72 \%$ & 6 & 132 & 156 & 117 & 171 & $5.21 \%$ \\
\hline 7 & 58 & 52 & 59 & 51 & $0.91 \%$ & 7 & 53 & 60 & 54 & 59 & $0.88 \%$ \\
\hline 8 & 139 & 148 & 152 & 135 & $4.53 \%$ & 8 & 134 & 146 & 129 & 151 & $1.79 \%$ \\
\hline 9 & 10 & 92 & 9 & 93 & $0.98 \%$ & 9 & 12 & 95 & 10 & 97 & $1.87 \%$ \\
\hline 10 & 102 & 211 & 112 & 201 & $3.19 \%$ & 10 & 51 & 56 & 48 & 59 & $2.80 \%$ \\
\hline 11 & 231 & 70 & 216 & 85 & $4.98 \%$ & 11 & 105 & 216 & 115 & 206 & $3.12 \%$ \\
\hline 12 & 47 & 66 & 42 & 71 & $4.42 \%$ & 12 & 235 & 77 & 221 & 91 & $4.49 \%$ \\
\hline 13 & 35 & 99 & 32 & 102 & $2.24 \%$ & 13 & 49 & 79 & 55 & 73 & $4.69 \%$ \\
\hline 14 & 51 & 80 & 53 & 78 & $1.53 \%$ & 14 & 38 & 97 & 34 & 101 & $2.96 \%$ \\
\hline 15 & 39 & 108 & 37 & 110 & $1.36 \%$ & 15 & 71 & 46 & 76 & 41 & $4.27 \%$ \\
\hline Tota1 & 1856 & 1385 & 1883 & 1358 & $\mathbf{0 . 8 3} \%$ & Total & 1891 & 1329 & 1857 & 1363 & $1.06 \%$ \\
\hline
\end{tabular}

\section{The Actual Data Validation} ODs.

Step1: obtain passengers travel time distribution from

Dawanglu-Yonghe Temple passenger travel time distribution based on AFC data is shown in Fig.4-3.

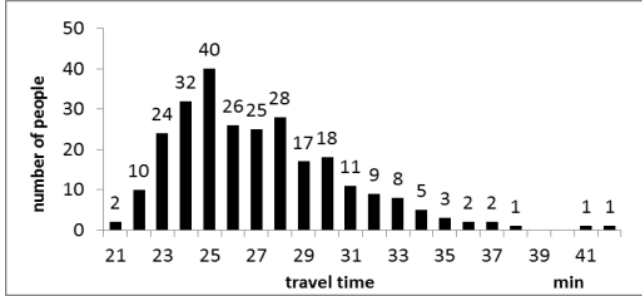

Figure 4-3. Dawanglu - Yonghe Temple Passenger Travel Time Distribution

Step2: calculating the parameters of normal distribution function of route 1 and route 2 .

Calculating the actual data using the algorithm mentioned in Chapter 3. Dividing normal distribution function of two routes according to passengers travel time distribution from ODs since passengers travel time distribution can't be divided into two portions based on actual data. Solving $\int_{m}^{n} \varphi_{T 1}(t) d t$ and $\int_{m}^{n} \varphi_{T 2}(t) d t$ in the formula by using NORMDIST function.

Step3: Calculating the proportion of the two routes respectively is

$\mu_{2}=\left(1-\mu_{1}\right)$, by the formula (3-9), the probability of choosing route 1 is $\mu_{1}=0.795522$.

Step4: calculating the number of passengers with the two routes
The probability of the passengers' choice route 1 is $79.55 \%$, the total number is 277 , so the number of route 1 is 220 . The probability of the passengers' choice route 2 is $20.45 \%$, the number is 57 .

The Passenger route selection problem about Dawanglu - Yonghe Temple show the same results generally through the methods of simulation verification or the actual data. The actual situation is the s Turnstile of line 2 and line 5 in Yonghe Temple are separated, it is observed that the actual passenger distribution ratio is route 1 about 222, route 2 about 55 In the AFC system,

Therefore, The probability of the passengers' choice route 1 is $80.14 \%$, The probability of the passengers' choice route 2 is $19.86 \%$, deviation is $0.72 \%$, Compared with the results in this paper, the difference is accepted, in accord with the actual situation, So the correctness, accuracy and applicability of the method are verified.

\section{CONCLUSIONS}

The paper not only structures multi-route ticket revenue clearing model using Automated Fare Collection Data by dividing passenger travel time, but validates the availability and accuracy of the model by the record of transactions at the entry and exit and simulation methods. These created a new idea for enhancing the accuracy of ticket revenue clearing.

\section{REFERENCES}

[1] Si Bingfeng, Mao Baohua, Liu Zhili. Passenger flow assignmentmodel for urban railway traffic network under the condition of seamless exchange $[\mathrm{J}]$. Journal of China Railway Society,2007,29(6):12-18.

[2] LUO Qin. Theoryand simulationanalysis ofpassenger flow distribution based on network operation for urban masstransit[D]. Shanghai:Tongji University,2009.

[3] SUN Yanshuo, XU Ruihua. Estimation of Rail Transit Passenger Route Using Automated Fare Collected Data[J]. Traffic and Transportation,2011,02:85-90.

[4] LIU Jianfeng, SUN Fuliang, BAI Yun. Passenger flow route assignment model and algorithm for urban rail transit network[J]. Journal of Transportation Systems Engineering and Information Technology,2009,9(5):131-133.

[5] QIAO Ke, ZHAO Peng, QIN Zhipeng. Route choice based on passenger classification for the urban mass transit network[J]. Journal of Beijing JiaoTong University,2012,36(6):102-106.

[6] Mao Baohua, Si Bingfeng, Liu Zhili. Urban Railway Traffic Network Management and TollAllocation:Theory andApplication[M]. Beijing: Science Publishing House,2007.

[7] GAO Shengguo, WU Zhong. Modeling Passenger Flow Distribution Based onTravel Time of Urban Rail Transit[J]. Journal of Transportation Systems Engineering and Information Technology,2011,11(6):125-130.

[8] XU Ruihua, LUO Qin, GAO Peng. Passenger Flow Distribution Model and Algorithm for Urban Rail Transit Network Based on Multi-route Choice[J]. Journal of the China Railway Society,2009,3(2):110-114.

[9] WEI Qiang, XIE Zongyi, ZHU Renrong. Fare clearing algorithm based on probabilistic model for urban rail transit[J]. Urban Mass Transit,2009,9:43-51.

[10] WU Xiangyun, LIU Canqi. Traffic Equilibrium Assignment Model Specially for Urban Railway Network [J]. Journal of Tongji University(Natural Sciece),2004,32(9): 1158-1162. 